\title{
Imagerie numérique par transparence : contrôle non destructif et imagerie dynamique
}

\author{
G. Bayon
}

Laboratoire de Support aux Programmes d'Irradiations, Départements des Réacteurs et Services Nucléaires, Direction de l'Énergie Nucléaire, Centre de Saclay, 91191 Gif-sur-Yvette, France

\section{1- Contrôles non destructifs et neutronographie}

2- Sources de neutrons utilisables en imagerie

\section{3- Détecteurs}

\section{4- Applications industrielles}

5- Imagerie numérique

6- Visualisation dynamique

7- Exemples d'applications

8- Conclusions

\section{Neutronographie et radiographie aux neutrons}

\section{Introduction}

La neutronographie est une technique de contrôle non destructif analogue dans son principe à la radiographie aux rayons $X$. Un faisceau de neutrons mis en forme à partir d'une source est utilisé pour fournir une image par transparence de l'objet examiné. Dans la suite de ce chapitre, on explique pourquoi cette technique est restée marginale dans l'arsenal des moyens d'examens non destructifs en rappelant l'historique du développement de la technique.

\section{Historique}

Les premières études sur l'ímagerie par transmission de neutrons ont été faites à Berlin entre 1938 et 1944 en utilisant un petit accélérateur permettant de générer des neutrons. Les flux disponibles étant très faibles, les images obtenues présentaient une qualité médiocre. C'est en utilisant le réacteur expérimental BEPO du centre d'études nucléaires d'Harwell (GB) que Twelis et Derbishyre ont produit les premières images neutronographiques en 1956. Au début des années 1960 , de nombreuses équipes de recherche furent en charge de développer les méthodes et rechercher des applications à ce nouveau moyen de contrôle non destructif. Les programmes de développement pour rechercher des sources neutroniques fiables et performantes débutèrent également sans trouver toutefois de solutions acceptables pour répondre aux contraintes d'un contrôle de production. La plupart des installations ayant une vocation industrielle sont aujourd'hui installées auprès d'un réacteur nucléaire expérimental dont l'imagerie par neutrons est une des activités qui y sont pratiquées. 


\section{Principe}

Une installation de neutronographie peut être schématisée par le dessin ci-dessous :

\section{Source de neutrons}

\section{Filtre Détecteur}

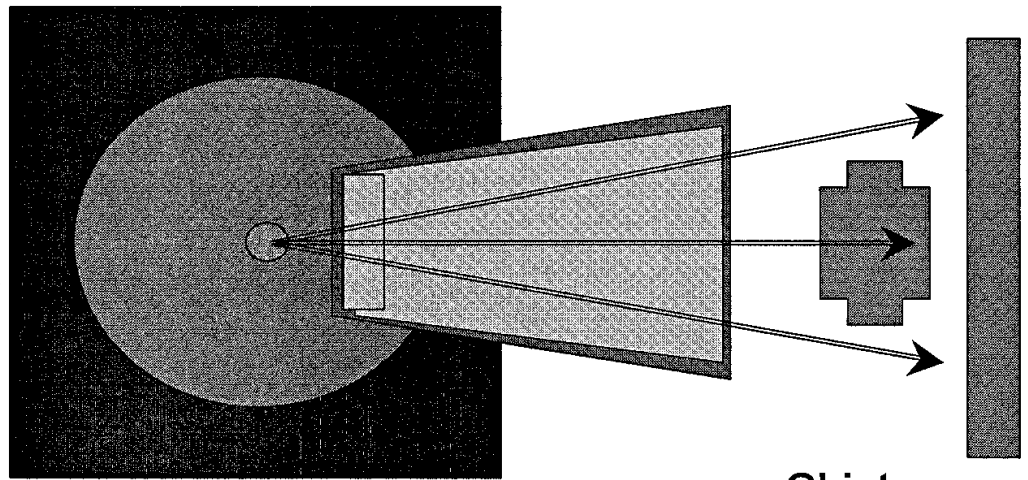

Objet

\section{Protection biologique \\ Modérateur Collimateur}

La source de neutrons est placée dans un milieu modérateur destiné à abaisser l'énergie des neutrons qui sont produits. A une distance calculée de cette source est disposée la fenêtre d'entrée du collimateur qui met en forme la faisceau. Sur cette figure, on a représenté un collimateur de forme divergente. Le filtre est un matériau qui fait barrage au rayonnement gamma pouvant accompagner l'émission neutronique. Le collimateur comporte des parois internes absorbant les neutrons et un gaz de remplissage transparent aux neutrons ( $\mathrm{He}$ ). A la sortie du collimateur, les neutrons interagissent avec l'objet en examen, ceux qui sont transmis à travers celui-ci sont absorbés par le détecteur pour former l'image.

\section{Sources neutroniques}

Les caractéristiques d'une source de neutrons performante, utilisable à des fins de contrôle sont les suivantes :

- Haut débit de fluence neutronique

- Faible pollution gamma

- Coût d'exploitation acceptable

- Radioprotection maîtrisée

- Manœuvrabilité

Le tableau ci-dessous regroupe différentes configurations ayant déjà été utilisées pour des applications de contrôle industriel. Un caractère commun à toutes les options est le coût d'achat et de maintenance très élevé par rapport à ceux des sources de rayons $X$. Ce handicap explique à lui seul pourquoi la neutronographie est restée une technique de contrôle non destructif marginale bien que ses applications potentielles soient au moins aussi étendues que celles de la radiographie. La définition d'une source destinée à l'imagerie est un compromis subtil prenant en compte de nombreux paramètres. Le type d'objet que l'on souhaite contrôler influe fortement sur les choix. Ainsi, le contrôle in-situ d'aéronefs sur une aire de maintenance impose d'utiliser une source transportable. Les exigences de masse seront alors beaucoup plus importantes que pour une installation fixe. 


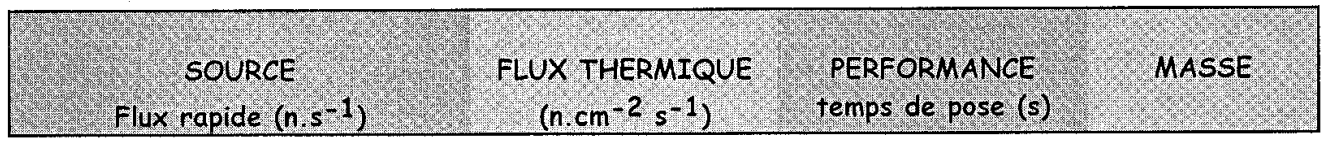

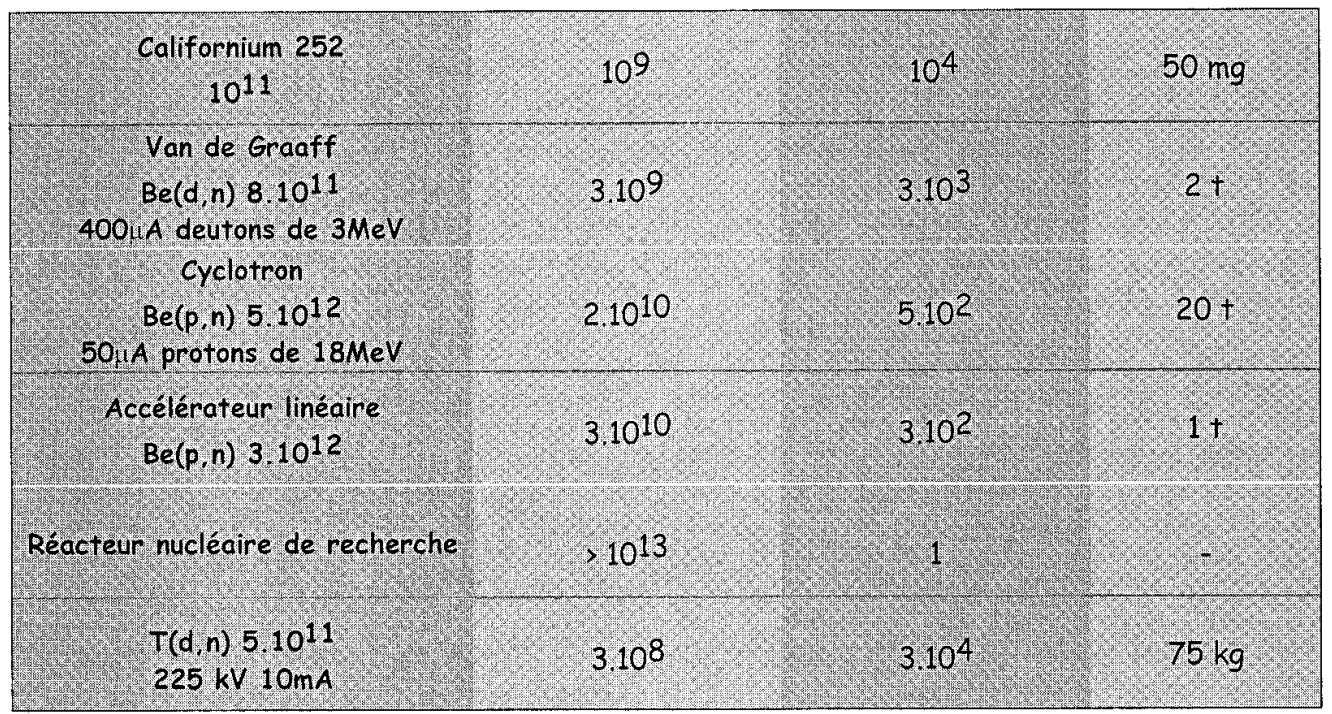

Toutes les sources neutroniques émettent un spectre de neutrons rapides qui est très rarement adapté pour faire de l'imagerie (voir le paragraphe consacré aux interactions neutrons/matière). Il convient alors de ralentir les neutrons dans un milieu appelé modérateur entourant la source rapide. Ce procédé entraîne malheureusement des pertes considérables d'intensité car le modérateur n'est pas totalement transparent aux neutrons et des captures parasites s'y produisent toujours. Dans le modérateur, les neutrons sont ralentis par chocs successifs accompagnés de transferts d'énergie aux atomes du milieu. Pour ce faire, les noyaux légers, en particulier d'hydrogène, sont souvent utilisés. On peut comparer des neutrons dans un modérateur à un gaz sous faible pression. On montre en particulier que l'énergie la plus probable d'une population de neutrons en équilibre est proportionnelle à la température de ce modérateur. Les neutrons «thermiques 》 dont on parle ici sont des neutrons dans un modérateur à $20^{\circ} \mathrm{C}\left(\mathrm{E}_{\mathrm{pp}}=2,510^{-2} \mathrm{eV}\right)$.

Cet effet de modération, d'autant plus élevé que l'énergie initiale des neutrons est grande, est matérialisé par les différences importantes entre les deux premières colonnes du tableau. Les valeurs présentées sont typiques d'installations existantes. Le flux disponible au niveau de l'objet est encore réduit par la valeur du rapport de collimation $\mathrm{L} / \mathrm{D}$, paramètre important qui va conditionner la taille du plus petit défaut visualisable sur l'installation. 


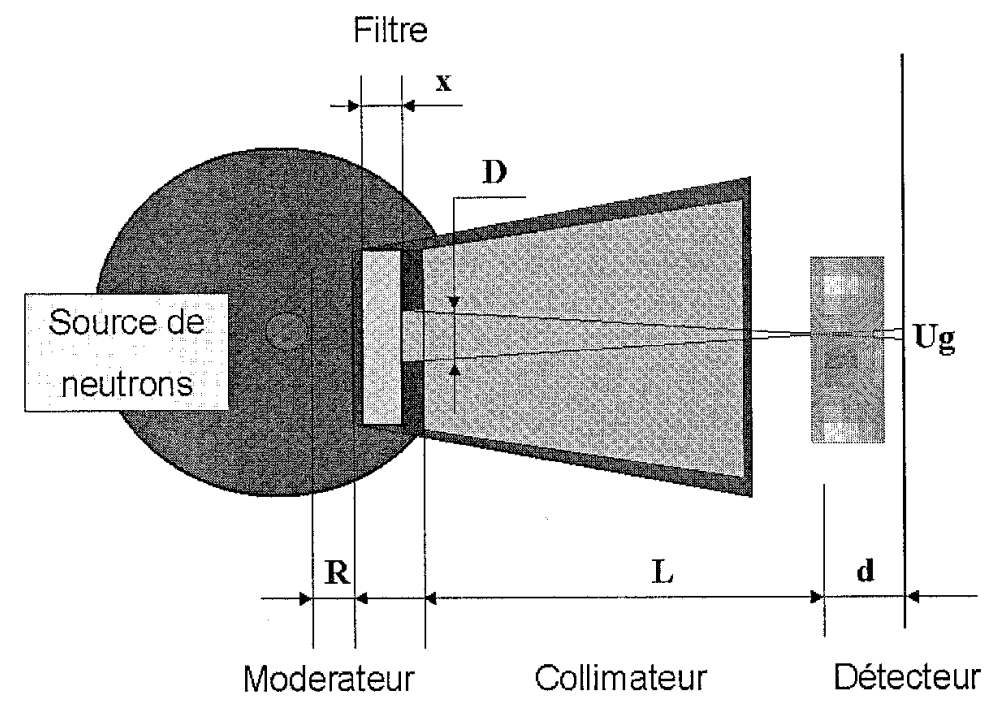

L est la distance qui sépare l'objet en cours de contrôle et la surface externe du masque de mise en forme de la fenêtre d'entrée du collimateur. D est la plus grande dimension de la surface d'entrée des neutrons. Cette surface peut être circulaire, rectangulaire ou carrée. Le flou géométrique caractéristique de l'installation Ug est en fait la projection d'un point matériel de l'objet sur la surface du détecteur d'image. Le flou est d'autant plus faible que $\mathbf{D}$ est petit et $\mathbf{L}$ est grand. Malheureusement, le flux de neutrons disponible au niveau de l'objet diminue également. Hors, le temps de pose pour réaliser le contrôle, inversement proportionnel au flux, est bien sûr un paramètre important en particulier sur la productivité de l'installation. On remarque également que la résolution géométrique des images (capacité à restituer des défauts de petites dimensions) est meilleure quand on réduit $\mathbf{d}$ au minimum. Pratiquement, le contact direct de l'objet et du système détecteur est recommandé.

Les réacteurs nucléaires de recherche, producteurs de flux neutroniques très importants sont donc les installations les mieux placées pour produire des neutronographies de très bonne qualité. De fait, ils occupent une très grande place parmi les installations ayant une vocation de contrôle non destructif de production. En revanche, ils ont au moins deux inconvénients majeurs :

- Obligation de déplacer les objets à contrôler auprès de l'installation (transports des pièces)

- Coût et contraintes d'exploitation hors de portée d'un industriel

Les autres sources utilisables offrent un intérêt limité et peuvent être envisagées uniquement quand les analyses ne peuvent être effectuées auprès d'un réacteur et quand une résolution dégradée est suffisante pour l'application envisagée. Il reste que ces sources sont très onéreuses à la fois à l'achat et en maintenance. Le budget correspondant est au moins dix fois supérieur à celui d'une installation par rayons $\mathbf{X}$ équipée d'un tube micro-foyer. Bien que l'imagerie neutronique soit une technique pouvant avoir de nombreuses applications potentielles, ce critère de coût la cantonne à un rôle marginal quand on la compare aux autres contrôles non destructifs. En fait, seules les applications ne pouvant pas être résolues par les méthodes traditionnelles font l'objet aujourd'hui de contrôles réguliers. Nous verrons ces applications dans la suite du texte.

Pour clore ce chapitre, on rappelle que la manipulation et l'utilisation de sources neutroniques de haute intensité imposent des règles strictes quant à la radioprotection des opérateurs au moins aussi contraignantes que pour les rayonnements électromagnétiques $\mathbf{X}$ et $\gamma$. 


\section{Interactions neutrons/matière}

Dans ce chapitre, on se contentera d'exposer le principe des phénomènes physiques et leurs conséquences en imagerie neutronique sans faire de développement scientifique. Pour aider à la compréhension globale des lecteurs initiés aux techniques de radiographie industrielle, une comparaison étroite entre le comportement des neutrons et des rayons $\mathbf{X}$ est présentée dans le tableau ci-dessous.

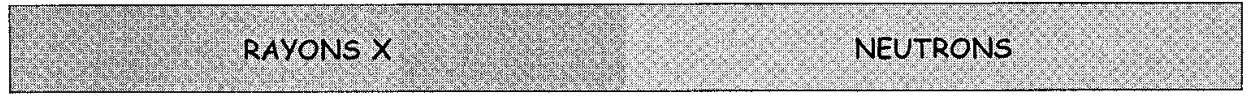

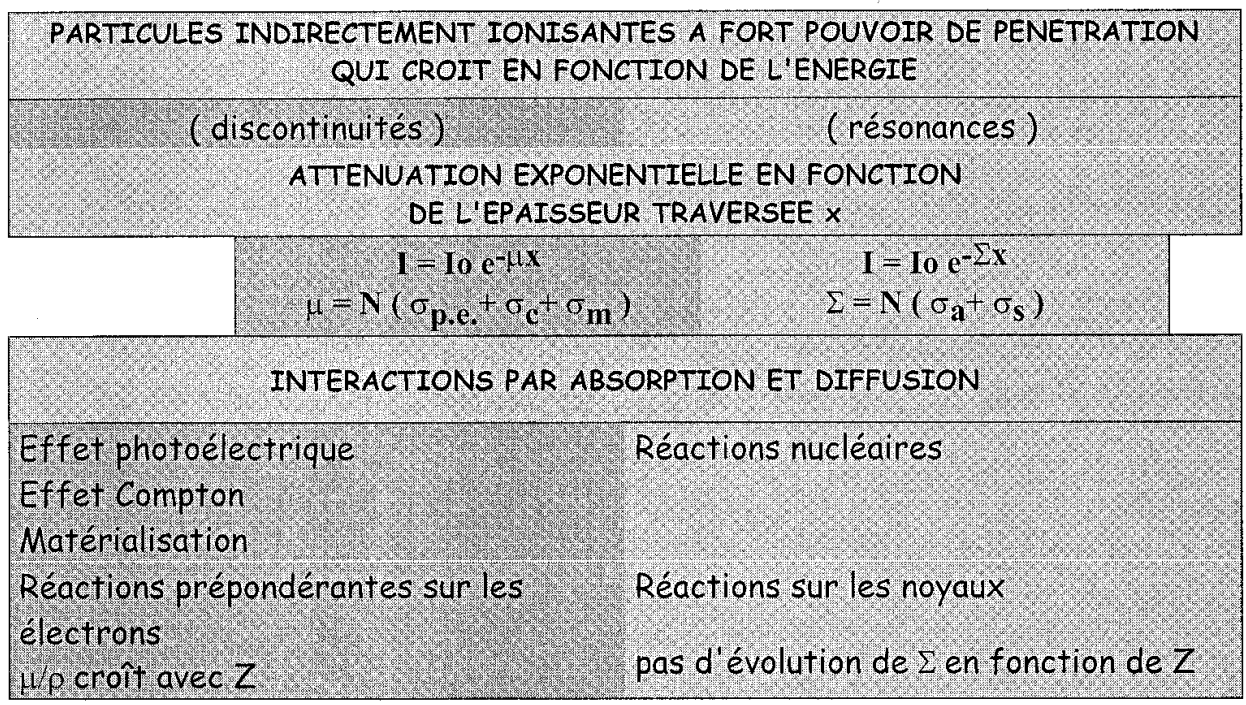

Certaines propriétés sont communes aux neutrons et aux rayonnements $X$ :

- Augmentation du pouvoir de pénétration du rayonnement en fonction de l'énergie exceptés les phénomènes de résonance et de discontinuité.

- Atténuation exponentielle de l'intensité du rayonnement en fonction de l'épaisseur de matériau traversée

- Interactions par absorptions et diffusions

En revanche, le caractère progressif de l'évolution des coefficients d'interaction en fonction du numéro atomique $Z$ que l'on observe pour les rayonnements électromagnétiques ne se retrouve pas pour les neutrons. Le diagramme ci-dessous reproduit les coefficients d'atténuation massique des différents éléments pour diverses énergies de rayons $\mathrm{X}$ et pour des neutrons thermiques. 


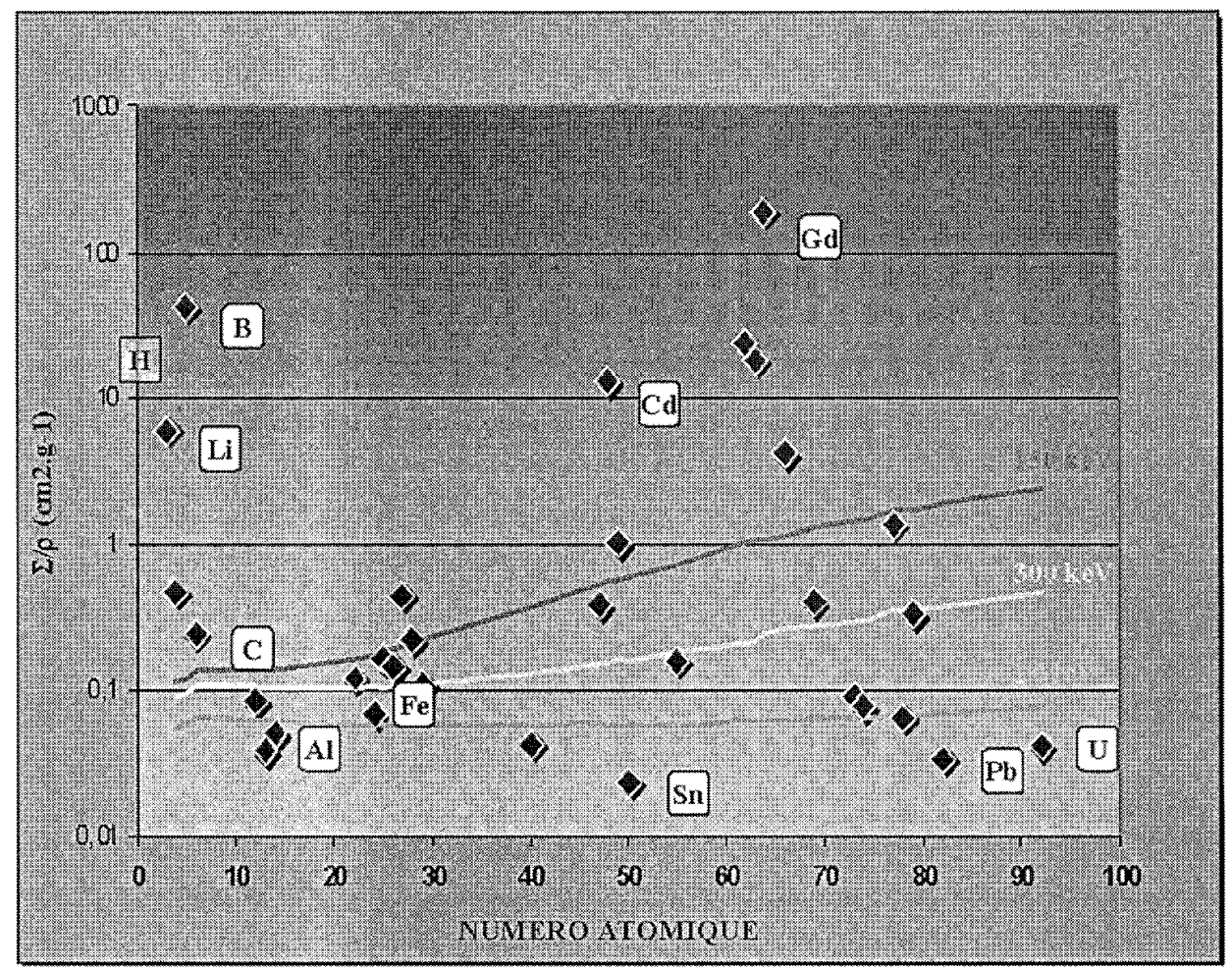

Ce diagramme permet de comprendre quelles sont les principales applications de l'imagerie neutronique :

- Visualisation de certains éléments « légers » $(\mathrm{H}, \mathrm{Li}, \mathrm{B})$ à travers ou en présence d'éléments métalliques qui eux sont transparents aux neutrons ( $\mathrm{Al}, \mathrm{Pb}, \mathrm{Fe}$ )

- Détection et quantification d'éléments particulièrement neutrophages ( $C d, G d, D y \ldots$ )

- Imagerie de matériaux très denses comme l'uranium

De plus, les isotopes d'un même élément ont en général des coefficients d'absorption différents. L'exemple le plus connu se rencontre sur l'uranium où l'isotope 235 présente une section efficace d'absorption plus élevée que celle de l'uranium 238. Ainsi, on peut également « quantifier » un enrichissement en prenant quelques précautions.

Il convient de préciser que l'irradiation aux neutrons peut provoquer l'apparition d'une radioactivité induite de l'objet contrôlé qui est d'autant plus importante que la fluence totale délivrée est élevée. Ce phénomène doit être bien pris en compte au moment de la manipulation des pièces après l'exposition aux neutrons.

\section{Détecteurs d'image}

Les neutrons étant des particules indirectement ionisantes, leur détection directe est assez difficile. Pour pallier ce handicap, on utilise très souvent un matériau à très haute section de capture neutronique pour avoir un bon rendement, cette capture s'accompagnant d'une émission secondaire de particules ionisantes pouvant exciter les détecteurs traditionnels : films photographiques, scintillateurs etc. Le choix d'un détecteur neutronique est également guidé par le type d'application envisagée. Le tableau ci-dessous regroupe les principaux couples de matériaux utilisés. 


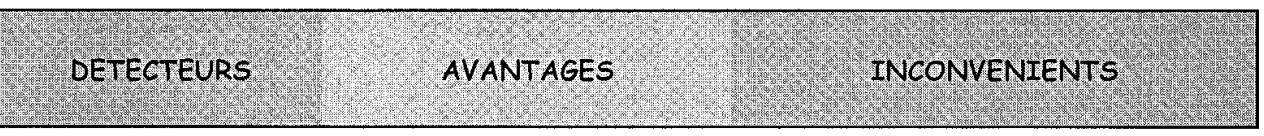

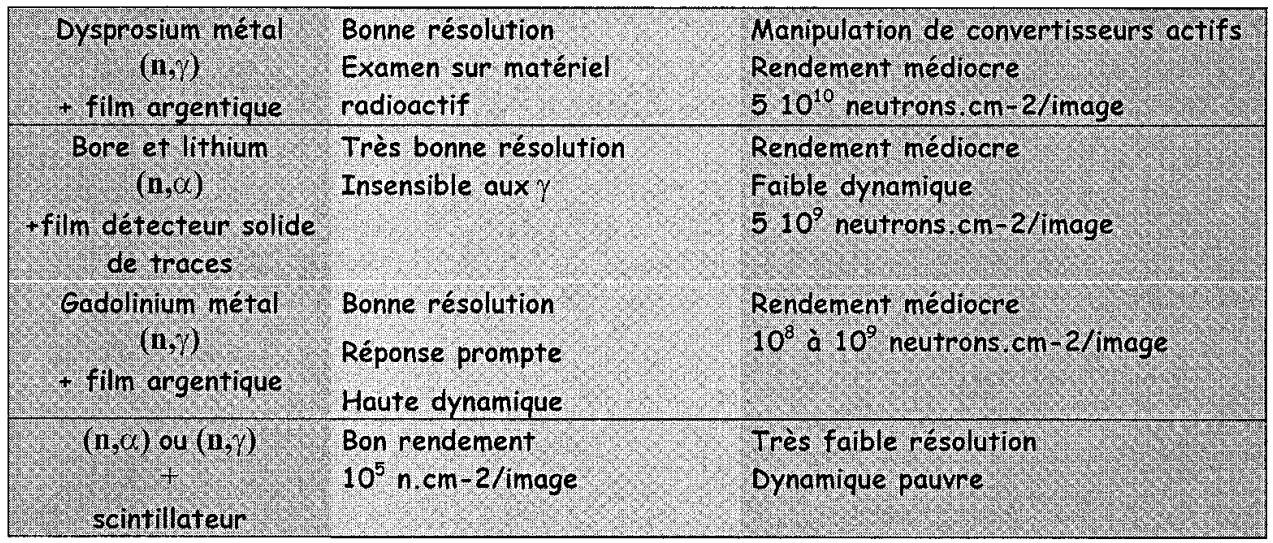

Le dysprosium est utilisé quand l'objet à contrôler est lui-même émetteur de rayonnements ou quand le faisceau neutronique est fortement pollué par un rayonnement $\gamma$ parasite. On effectue l'examen en deux étapes successives :

- Irradiation neutronique de l'objet et d'une feuille de dysprosium

- Exposition d'un film argentique par le dysprosium pendant la décroissance de celui-ci

Le couple très largement utilisé pour les examens de type industriel est une association d'une feuille de gadolinium et d'un film photographique associés dans une cassette à vide qui assure à la fois l'étanchéité à la lumière et le maintien mécanique du sandwich. Ce système détecteur permet d'obtenir des qualités d'images analogues aux meilleures radiographies traditionnelles. En revanche, le rendement de détection est assez faible et nécessite l'emploi de sources neutroniques de forte intensité. Cette solution est souvent utilisée sur les réacteurs nucléaires à l'aide d'un faisceau de neutrons convenablement filtré.

Le bore et le lithium produisent une particule alpha après absorption d'un neutron. Les alpha ayant un transfert linéique d'énergie très élevé provoquent des ruptures de chaînes macromoléculaires dans certains supports plastiques comme la nitrocellulose et autres détecteurs solides de traces. Ces films sont ensuite " développés 》 dans un bain de soude qui amplifie les traces et les rend visibles jusqu'à former une image. Ce couple peut également être utilisé pour contrôler des pièces radioactives. Le détecteur solide de traces est manipulable à la lumière mais il difficile à obtenir en grandes dimensions. Les convertisseurs émetteurs alpha sont dispersés au sein de milieu scintillant comme le sulfure de zinc pour former un détecteur neutronique ayant un très bon rendement mais en revanche la résolution de tels détecteurs est assez médiocre. Ils sont largement utilisés pour les systèmes de scopie (imagerie en temps-réel) ou le rendement de détection doit être absolument privilégié.

La plupart des détecteurs électroniques utilisés en imagerie par rayons $X$ peuvent également être mis en œuvre pour effectuer des images sous neutrons. Il suffit d'adjoindre un récepteur ayant une bonne section efficace neutronique et vérifier que les senseurs électroniques sont résistants à l'irradiation. On rappelle ici que le silicium transmute en phosphore sous irradiation aux neutrons et que cet effet peut entraîner la destruction rapide des semi-conducteurs.

Le développement des matériels et logiciels informatiques et la réduction de leur coût incitent de plus en plus à privilégier les techniques d'imagerie numérique directe au détriment du film photographique. Ces dernières années ont vu le développement de détecteurs photo-stimulables par laser en imagerie médicale et industrielle, ils sont maintenant utilisables avec des neutrons. La dynamique et la linéarité de réponse de ces détecteurs sont nécessaires pour envisager l'utilisation de logiciels de traitement d'images destinés à produire des résultats de coupes tomographiques ou des restitutions volumiques.

\section{Applications de l'imagerie par neutrons}

\section{Contrôle de production}

La grande majorité des applications de la neutronographie «industrielle » découlent de la forte interaction entre neutrons et noyaux d'hydrogène. Beaucoup de molécules en contiennent et l'imagerie par neutrons permet de les 
visualiser à travers des structures métalliques qui elles, sont transparentes aux neutrons. Historiquement, la première application industrielle en France a été pratiquée pour contrôler la continuité des explosifs dans les cordeaux détonants de transmission et de découpage utilisés en pyrotechnie spatiale. Trente ans après ces premiers essais, la neutronographie permet de contrôler la totalité de cette production utilisée sur les lanceurs spatiaux comme Ariane. Le but est de s'assurer qu'aucun défaut jugé comme critique ne subsiste dans l'explosif après la fabrication des produits. Typiquement, un défaut volumique de $1 / 10$ de millimètre peut être détecté à travers une enveloppe métallique en plomb ou en aluminium $(\sim 1 \mathrm{~mm})$ contenant l'explosif. Il n'existe pas d'autre technique de contrôle non destructif répondant à ce besoin en dehors de la neutronographie.

Sur le même principe, les neutrons sont capables de visualiser des collages (matériaux composites), des isolants en résine (composants électroniques), des joints organiques d'étanchéité, des films ou des capacités d'eau ou d'huile, des revêtements de surface (vernis, peinture), l'apparition de corrosion dans des alliages d'aluminium (hydroxydes), des résidus de combustion (cokéfaction). Loin d'être exhaustive, cette liste énonce des applications d'expertise ayant été menées en France.

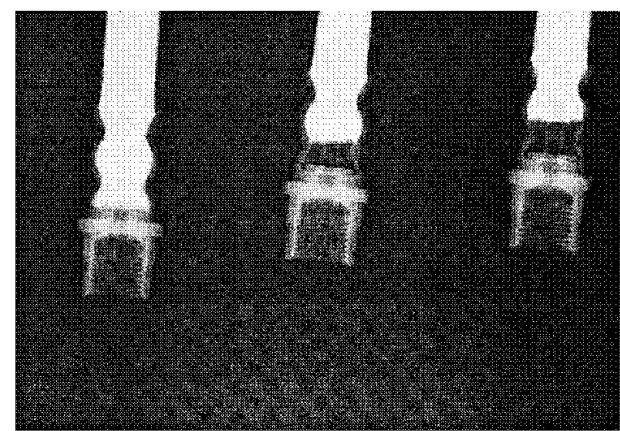

Neutronographie d'extrémités de flexibles de freins automobile : visualisation de jeux entre flexibles et embouts métalliques

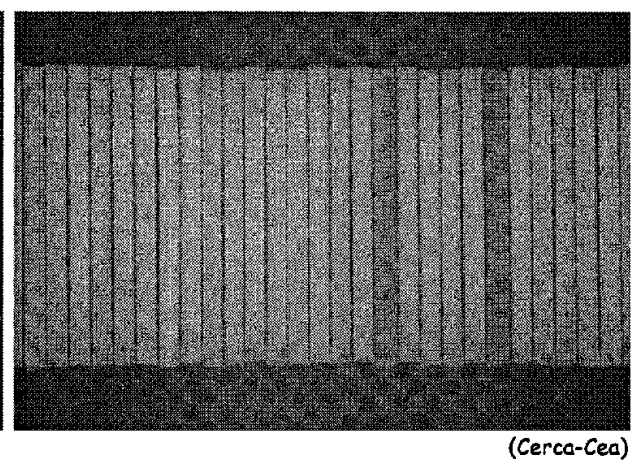

Neutronographie d'échantillons de plaquettes

d'aluminium borées : visualisation d'agglomérats et de défauts de chargement

Certains contrôles métallurgiques peuvent être traités en utilisant les neutrons spécialement quand un composé comme le bore, le lithium ou le cadmium est réparti dans une matrice métallique comme de l'acier ou un alliage d'aluminium. Pour des applications spécifiques du domaine nucléaire (poison consommable ou protection contre le risque de criticité), on peut cartographier la répartition et évaluer quantitativement la teneur de l'élément neutrophage en utilisant des étalons de géométrie identique et ayant des charges encadrant les spécifications de production.

Une application toujours très répandue consiste à contrôler le « décochage » des aubes de turbine fabriquées par moulage sous pression de superalliages à base nickel. Des noyaux de céramique sont utilisés pour reproduire les canaux de refroidissement des aubes. Après solidification, ces noyaux sont dissous par voie chimique. Les aubes fonctionnant dans des gammes de température très élevées, le refroidissement doit être optimal et des résidus de noyaux sont extrêmement dommageables en service. Le contrôle des aubes est effectué après imprégnation de celles-ci dans une solution de sulfate de gadolinium et rinçage. Les résidus de céramique adsorbent ce « révélateur » qui est ensuite facilement mis en évidence par imagerie neutronique.

\section{Contrôle d'expertise}

A l'inverse de ce qui a été énoncé précédemment, ces examens ne portent pas sur de grandes séries de production mais sur des prototypes, des échantillons d'études ou des pièces présentant un défaut déjà identifié et que l'on souhaite mieux caractériser. Le contrôle peut également valider des options de réalisation sur un nombre restreint d'échantillons. Le procédé une fois figé ne nécessite plus de contrôles systématiques.

De nombreuses études ont montré la capacité des neutrons à détecter de façon très précoce la corrosion de pièces de structure aéronautiques en alliages d'aluminium. Les hydroxydes formés apparaissent très aisément sur les clichés du fait de la concentration locale d'atomes d'hydrogène. 


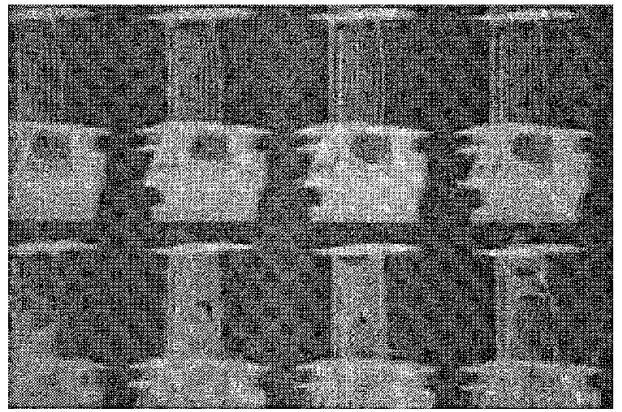

(Howmet Turbines LTD-Cea)

Neutronographie d'aubes de turbine en superalliage : visualisation de destruction de noyaux céramiques

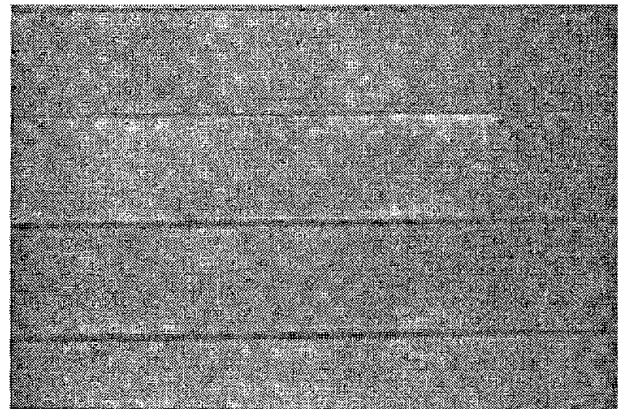

(EADS-Cea)

Neutronographie de plaquettes d'alliage d'aluminium : visualisation de corrosion créée en environnement marin

L'imagerie neutronique n'a pas pu s'imposer comme contrôle sur site pour des raisons multiples :

- Manque de performances des sources entrainant des temps de pause prohibitifs

- Radioprotection du personnel difficile à mettre en œuvre sur site de maintenance

- Difficulté d'automatisation des contrôles

- Expertise de l'opérateur pour le diagnostic de zones singulières etc.

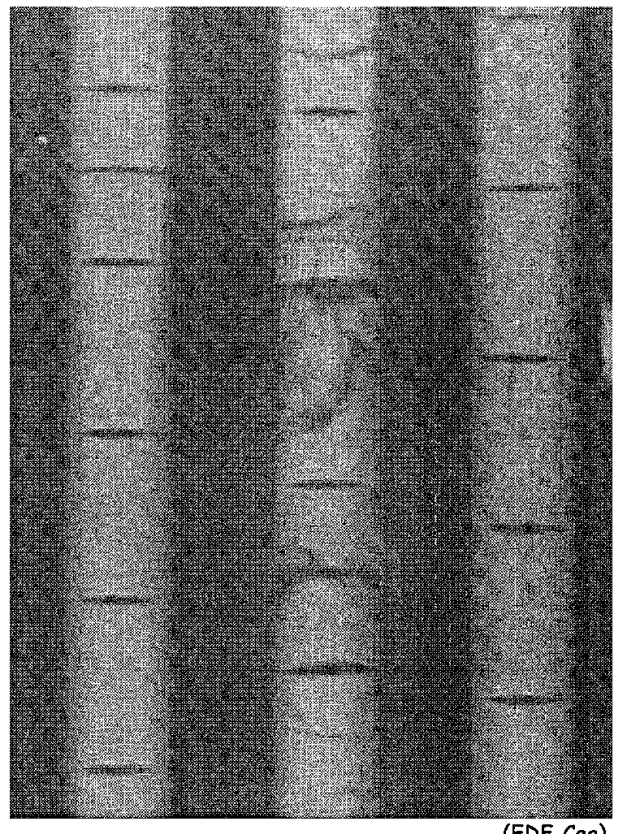

(EDF-Cea)

Neutronographie de crayons combustibles $\mathrm{UO}_{2}$ irradiés en centrale de puissance

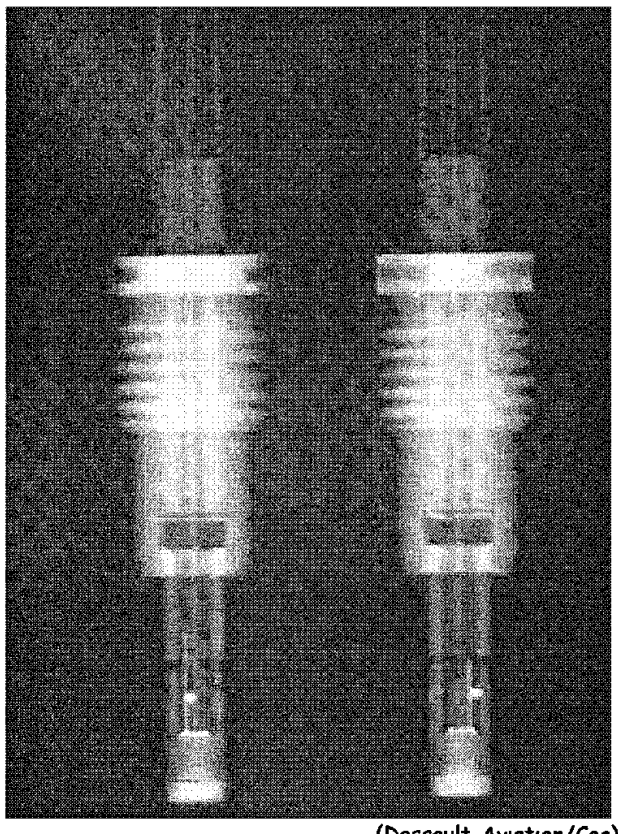

(Dassault-Aviation/Cea)

Neutronographie d'extrémités de lignes de transmissions de détonation ARIANE 5

\section{Matériaux irradiés}

Historiquement, la neutronographie a été et est toujours utilisée pour obtenir des images de matériaux fortement irradiants. La technique par transfert déjà exposée ci-dessus permet d'obtenir des informations qualitatives et parfois quantitatives sur les combustibles nucléaires et en particulier les modifications apportées par l'irradiation neutronique. 


\section{Imagerie dynamique}

L'enregistrement d'images neutroniques à la cadence vidéo impose d'utiliser des détecteurs à haut rendement. Le chapitre sur les imageurs a montré que le bore ou le lithium associé à un scintillateur constituait la solution la plus éprouvée à ce jour.

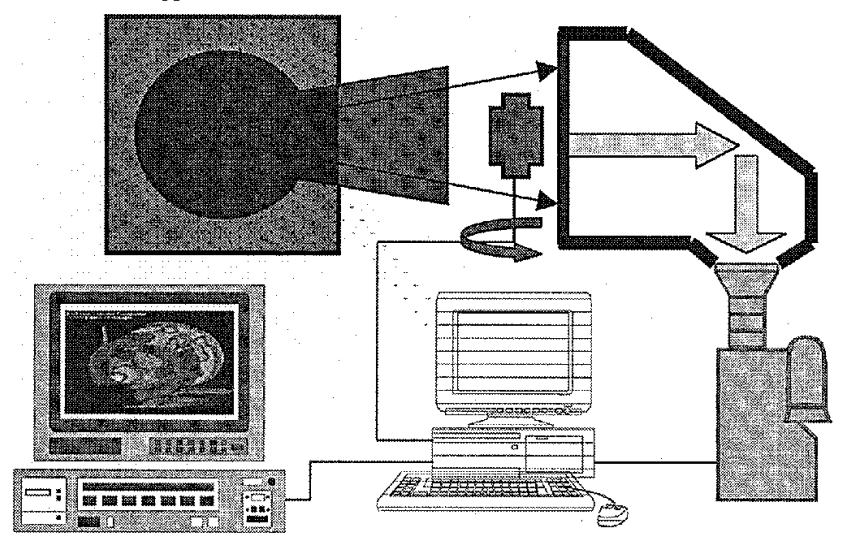

Dans le schéma reproduit ci-contre, la lumière émise par le scintillateur est recueillie par l'objectif de la caméra à haute sensibilité après un renvoi d'angle optique qui permet d'éviter l'irradiation directe de la caméra. Le signal vidéo est transmis via une carte d'acquisition et est enregistré sur un magnétoscope.

Les déplacements de l'objet, rotation et/ou translation sont gérés par le micro-ordinateur. L'utilisation d'algorithmes spécifiques permet de reconstituer des coupes tomographi-ques ou des reconstructions volumiques.

Historiquement, l'Imagerie neutronique en dynamique a fourni des renseignements intéressants pour la visualisation des écoulements diphasi-

ques par exemple dans des tubulures d'échangeurs ou de condenseurs. La quantification du taux de vide peut ainsi être obtenue en temps réel pendant le déroulement des essais. La répartition des lubrifiants dans différentes structures métalliques comme des carters moteurs ou boîte de vitesses a également été visualisée par imagerie neutronique. La circulation de fluides dans des milieux poreux est également quantifiable et a fait l'objet d'études particulières dans les domaines suivants:

- Croissance des racines de plantes en fonction de l'humidité du milieu

- Migration de polluants dans les sols

- Transfert d'eau dans des matériaux de construction etc.

(Total-Cea)

Les études portant sur le remplacement du Fréon dans les appareils de réfrigération domestique ont conduit les équipes de recherche du groupe Total à s'adresser au CEA pour tenter de visualiser le comportement en service des fluides frigorigènes exempts de CFC. La difficulté du programme résidait dans le développement d'une huile de synthèse économique ayant une bonne miscibilité avec le R134A. Cette propriété est nécessaire au bon fonctionnement des groupes à circuit unique fermé. Sur l'image neutronique ci-contre, traitée en pseudo couleurs, on distingue une l'huile

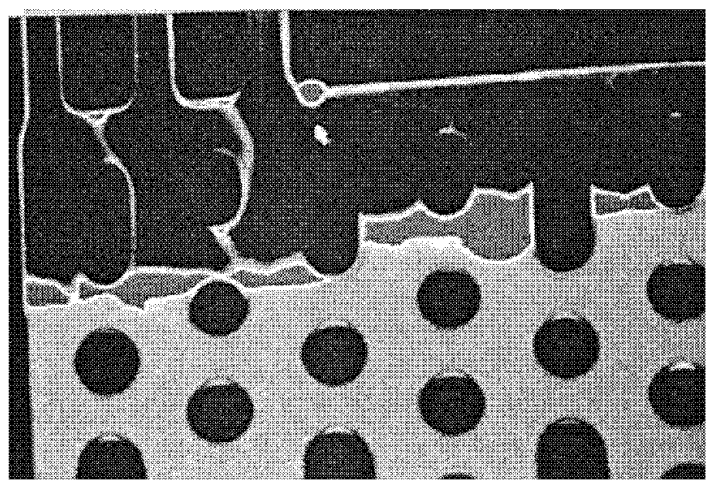
minérale (en rouge) qui surnage au dessus du fluide liquide frigorigène (en vert). Ce contraste est restitué par les neutrons à cause de la différence de concentration en nombre d'atomes d'hydrogène par unité de volume qui est beaucoup plus élevée pour l'huile. La neutronographie en dynamique a permis de fournir des renseignements complémentaires aux essais d'endurance. En particulier, des phénomènes inattendus ont été visualisés pendant le remplissage de l'évaporateur lors de certaines phases critiques de fonctionnement comme le démarrage à froid du compresseur. 


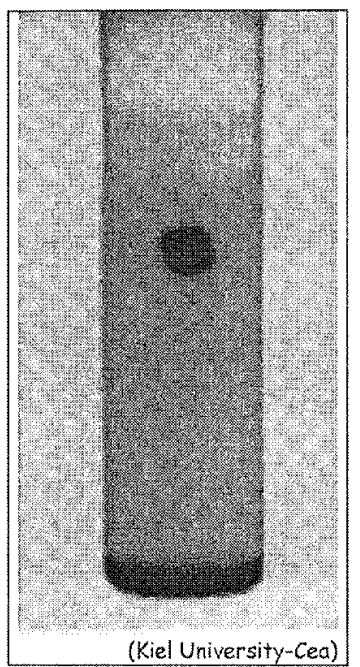

Une autre application spectaculaire qui fait l'objet de campagnes de mesure actuellement à Saclay est dérivée du principe de viscosimètre à bille. D'après la loi de Stoke

$$
\eta=2 C_{F} g r^{2}(\rho \text { sphère }-\rho \text { milieu }) / 9 V \text { limite }
$$

Où $\eta$ est la viscosité dynamique (grandeur recherchée)

$C_{F}$ est un facteur de correction

$r$ le rayon de la sphère

$\rho_{i}$ la masse volumique du milieu $\mathrm{i}$

$V_{\text {limite }}$ est la vitesse maximale stabilisée au cours de la descente

Cette loi est appliquée pour connaître la viscosité de silicates fondus a haute température $\left(\sim 1800^{\circ} \mathrm{C}\right)$. On utilise un four électrique à parois transparentes aux neutrons et une bille réfractaire opaque aux neutrons. Les séquences de descente de la bille dans le milieu fondu sont enregistrées en vidéo et permettent avec une calibration en distance de mesurer la vitesse limite de descente. L'illustration ci-contre montre une image vidéo où on voit la bille descendre dans l'échantillon liquide. Les chercheurs en science de la terre sont particulièrement intéressés par ces mesures qui leur permettent par exemple de modéliser les phénomènes volcaniques.

\section{Conclusions}

L'imagerie par neutrons est utilisée pour répondre à des besoins divers depuis le contrôle non destructif de production des fabrications pyrotechniques jusqu'à des mesures fines à vocation scientifique. Ce panel est cependant limité par absence de sources neutroniques suffisamment puissantes et bon marché. Toutes les voies industrielles explorées jusqu'à présent ont abouti à des prototypes de sources ne trouvant par de " marché commercial ». Les réacteurs nucléaires de recherche demeurent les meilleures installations pour le développement des applications potentielles. 